\title{
Valproic acid enhances the antileukemic effect of cytarabine by triggering cell apoptosis
}

\author{
NAN LIU $^{1 *}$, CHEN WANG $^{2 *}$, LIBING WANG $^{1 *}$, LEI GAO $^{1}$, HUI CHENG $^{1}$, \\ GUSHENG TANG ${ }^{1}$, XIAOXIA HU ${ }^{1}$ and JIANMIN WANG ${ }^{1}$ \\ ${ }^{1}$ Institute of Hematology, ${ }^{2}$ Department of Traditional Chinese Medicine, Changhai Hospital, \\ The Second Military Medical University, Shanghai 200433, P.R. China
}

Received October 9, 2015; Accepted March 23, 2016

DOI: $10.3892 /$ ijmm.2016.2552

\begin{abstract}
Acute myeloid leukemia (AML) is an aggressive clonal malignancy of hematopoietic progenitor cells with a poor clinical outcome. The resistance of leukemia cells to contemporary chemotherapy is one of the most formidable obstacles to treating AML. Combining valproic acid (VPA) with other anti-leukemic agents has previously been noted as a useful and necessary strategy which can be used to specifically induce anticancer gene expression. In the present study, we demonstrated the synergistic antileukemic activities between VPA and cytarabine (Ara-C) in a retrovirus-mediated murine model with MLL-AF9 leukemia, three leukemia cell lines (THP-1, K562 and HL-60) and seven primary human AML samples. Using RT-qPCR, we noted that the combination of VPA and Ara-C significantly upregulated $B A X$ expression and led to the arrest of leukemia cell proliferation, sub-G1 DNA accumulation and cell apoptosis, as demonstrated by flow cytometric analysis. Significantly, further experiments revealed that knockdown of $B A X$ expression prevented VPA and Ara-C-induced cell apoptosis in THP-1 cells. The results of our present study demonstrated the synergistic antileukemic effect of combined VPA and Ara-C treatment in AML, and thus we suggest that VPA be used an alternative treatment for AML.
\end{abstract}

\section{Introduction}

Acute myeloid leukemia (AML) is a heterogeneous group of malignancies which is characterized by the uncontrolled proliferation, abnormal survival and maturation arrest of neoplastic hematopoietic stem cells. AML is the most common type of adult acute leukemia (1). According to data from published

Correspondence to: Dr Jianmin Wang or Dr Xiaoxia Hu, Institute of Hematology, Changhai Hospital, The Second Military Medical University, 168 Changhai Road, Shanghai 200433, P.R. China

E-mail: jmwang@medmail.com.cn

E-mail: huxiaoxia@medmail.com.cn

*Contributed equally

Key words: valproic acid, histone deacetylase inhibitor, acute myeloid leukemia, cytarabine studies, $50-80 \%$ of adult patients can achieve complete remission (CR) after intensive chemotherapy, whereas 40-50\% of patients who achieve CR eventually relapse $(2,3)$. AML predominantly affects elderly adults, with a median age at diagnosis of 68 years (4). Unfortunately, most elderly patients cannot tolerate the side-effects of intensive chemotherapy and tend to be more resistant to standard chemotherapy drugs: only $30-50 \%$ achieve complete remission, and $<10 \%$ are long-term survivors $(4,5)$. Thus, the outcome for elderly patients is disappointing, and is markedly inferior to that obtained for younger patients. Therefore, there is an urgent need to develop a novel therapy with low toxicity and high efficacy.

Traditionally, AML has been considered the result of genetic alterations, but experimental evidence has demonstrated that epigenetic modifications are important in the development and maintenance of leukemia cells (6). Histone deacetylase inhibitors (HDACi) have the potential to exert antitumor effects (7) and thus represent an important therapeutic alternative for cases of AML $(8,9)$. HDACi exert their anticancer effects through a variety of mechanisms, including stress-related pathway activation, protective pathway inhibition, death receptor upregulation, ceramide generation, heat shock protein accumulation, as well as oxidative damage collapse (10). Valproic acid (VPA) is a short-chain fatty acid that has been used as an anticonvulsant for the past 30 years. It has become evident that VPA also exerts antitumor effects, which has led to preclinical reserach showing that VPA induces a loss of proliferative capacity and promotes the differentiation of several tumor cell types (11). VPA also regulates tumor growth, thus affecting different cell properties, including proliferation, differentiation, tumor metastasis, tumor immunogenicity and angiogenesis; one important finding is that VPA functions as an HDACi, possibly by binding to the catalytic centre of HDACs (12). Several previous studies have shown that VPA has a broad impact on AML blast cells by inhibiting HDAC class I activity, and this effect is usually accompanied by increased levels of histone $\mathrm{H} 3$ and $\mathrm{H} 4$ acetylation (7-10). Cytarabine (Ara-C) is a chemotherapeutic drug that is used alone or in combination with other traditional antileukemic regimens to treat different forms of AML. As a deoxycytidine analog, this antimetabolite drug is incorporated into human DNA, consequently killing leukemia cells by interfering with DNA and RNA synthesis (13). 
In the present study, we hypothesized that VPA synergizes with Ara-C, resulting in an enhanced antileukemic effect in AML. This synergy upregulated $B A X$ expression and led to leukemia cell proliferation arrest, sub-G1 DNA accumulation and cell apoptosis. We thus propose that VPA is an alternative treatment choice in the treatment of AML.

\section{Materials and methods}

Clinical samples. Diagnostic bone marrow samples $(\mathrm{n}=7)$ from patients with de novo AML were obtained from the Institute of Hematology, Changhai Hospital (Shanghai, China). BM samples were selected from cases with sufficient cell numbers (minimum $5 \times 10^{6}$, blast percentage $>75 \%$, viability $>85 \%$ ) Patient characteristics are summarized in Table I. Mononuclear cells were purified by standard Ficoll-Hypaque density centrifugation (GE Healthcare, Logan, UT, USA) in a GH-3.7 rotor at $900 \mathrm{x}$ g for $30 \mathrm{~min}$ at $18-20^{\circ} \mathrm{C}$ with no brake. Informed consent was provided according to the Declaration of Helsinki. Sample handling and data analysis protocols were approved by the Human Investigation Committee of Second Military Medical University (Shanghai, China).

Cell culture. The THP-1 (AML-M5 cells carrying $\mathrm{t}(9 ; 11)$ (p22;q23) and expressing $M L L-A F 9$ ) cell line was a gift from the Chinese Academy of Sciences (Beijing, China); the K562 (a $B C R$ - $A B L$-positive CML blast crisis patient cell line) and HL-60 (AML-M3) cell lines were continuously passaged in our laboratory. We cultured the cell lines in RPMI-1640 with $10-20 \%$ fetal bovine serum (HyClone, Logan, UT, USA) in a humidified atmosphere at $37^{\circ} \mathrm{C}$ containing $5 \% \mathrm{CO}_{2} / 95 \%$ air.

Mice. C57BL/6J (CD45.2+) mice were purchased from the Institution of Zoology of the Chinese Scientific Academy (Shanghai, China). B6.SJL-PtprcaPepcb/BoyJ mice (B6.SJL, CD45.1 $1^{+}$were obtained from Dr Tao Cheng (State Key Laboratory of Experimental Hematology, Tianjin, China) and maintained in our animal facility. All mice were maintained in specific pathogen-free conditions. All animal protocols were approved by the Institutional Animal Care and Use Committees of all institutions participating in this study.

Mouse model of leukemia and in vivo treatment studies. The retrovirus vector containing cDNA-encoding $M L L-A F 9$ was kindly provided by Dr Tao Cheng (Chinese Academy of Medical Sciences, Tianjin, China). The plasmid (MSCV-MLL-AF9-IRES-GFP) was co-transfected into the 293 T cell line with pCMV-VSV-G and pKAT using Lipofectamine 2000 (Invitrogen, Carlsbad, CA, USA). Supernatant was harvested 48 and $72 \mathrm{~h}$ after transfection. Lineage negative $\left(\mathrm{Lin}^{-}\right)$cells from the bone marrow (BM) of female C57BL/6 mice $\left(\mathrm{CD} 45.2^{+}\right)$were enriched with biotin-conjugated lineage antibodies [CD3 (RM3415-3), CD4 (RM2515-3), CD8 (RM2215-3), CD11b (Mac-1, RM2815-3), Gr-1 (Ly-6G, RM3015-3), CD45R (B220, RM2615-3), Ter-119 (MTER 15-3); all from Caltag Laboratories, Burlingame, CA, USA], and selected with microbead-conjugated streptavidin (Miltenyi Biotec GmbH, Bergisch Gladbach, Germany) according to the manufacturer's instructions. Lin cells were re-enriched for expression of Sca-1 using the EasySep murine Sca-1 positive selection kit (Stem Cell Technologies, Vancouver, BC, Canada). Transduction of Lin-Sca-1 positive (Lin-Sca- $1^{+}$) cells with $M L L-A F 9$ plasmid was performed as previously described with minor modifications (14). The transduction efficiency was measured by flow cytometric analysis.

Mouse BM cells were obtained by flushing ilia, femurs, and tibias as previously described (14). Lin $^{-}$cells (CD45.2 ${ }^{+}$) were isolated from the $\mathrm{BM}$ of $\mathrm{C} 57 \mathrm{BL} / 6 \mathrm{~J}$ mice at the age of 6 to 8 weeks and subsequently transduced with the MSCV-MLL-AF9-IRES-GFP vector. A total of $10^{6}$ transduced cells were transplanted into lethally irradiated recipients (9.5 Gy, C57BL/6J). Primary recipient mice were sacrificed at terminal disease stage under anesthesia. Leukemia cells were harvested from enlarged spleens and filtered through 100-mm cell strainers (BD Falcon, Franklin Lakes, NJ, USA) to obtain single-cell suspensions. For in vivo studies, $1 \times 10^{6}$ leukemic cells were transplanted by tail vein injection into 6-8-week-old non-irradiated B6.SJL recipient mice $\left(\mathrm{CD} 45.1^{+}\right)$. Chemotherapy was initiated 11 days after transplantation, which correlated with approximately $10 \%$ bone marrow infiltration, as assessed by flow cytometric analysis (FACScan; BD Biosciences, San Jose, CA, USA). Leukemic mice were randomized into four groups and treated for 5 consecutive days by intraperitoneal injection with one of four treatments. The first group (control) received $100 \mu \mathrm{l}$ PBS only, the second received VPA (400 $\mathrm{mg} / \mathrm{kg}$, every $12 \mathrm{~h}$ ) and the third received Ara-C (100 mg/kg, every $24 \mathrm{~h})$. The fourth group received VPA (400 mg/kg, every $12 \mathrm{~h}$ ) and Ara-C (100 mg/kg, every 24 h). Survival curves were plotted using Kaplan-Meier estimates. Statistical analysis was performed using SPSS software (version 11.0; SPSS Inc., Chicago, IL, USA).

Drugs. VPA was purchased from Sigma-Aldrich (St. Louis, MO, USA) and Ara-C was purchased from Pharmacia Italia SPA (Nerviano, Italy).

In vitro cytotoxicity assays. In vitro Ara-C and VPA cytotoxicities of leukemia cell lines and diagnostic blasts were measured using a Cell Counting kit-8 (CCK-8; Dojindo Laboratories, Kumamoto, Japan) assays. Median inhibitory concentrations $\left(\mathrm{IC}_{50}\right)$ were determined as the drug concentrations required to inhibit proliferation in $50 \%$ of the cells compared with untreated control cells. The extent and effect of VPA and Ara-C cytotoxic interactions were evaluated as described previously $(15,16)$. The combination index $(\mathrm{CI})$ was calculated as follows: $\mathrm{CI}=[(\mathrm{D}) 1 /(\mathrm{Dx}) 1]+[(\mathrm{D}) 2 /(\mathrm{Dx}) 2]$. At $50 \%$ inhibition, (Dx)1 and (Dx)2 were the concentrations of Ara-C and VPA when administered individually; (D)1 and (D)2 were the concentrations of Ara-C and VPA when combined. $\mathrm{CI}<1, \mathrm{CI}=1$, and $\mathrm{CI}>1$ indicate synergistic, additive and antagonistic effects, respectively. The $\mathrm{IC}_{50}$ values are presented as the means \pm standard error of at least three independent experiments.

Apoptosis assay. Cell apoptosis was measured by staining with FITC-conjugated Annexin V/propidium iodide (PI) (eBioscience, San Diego, CA, USA) and determining with a flow cytometer according to the manufacturer's instructions as described previously (17). Apoptotic events were recorded as a combination of Annexin $\mathrm{V}^{+} / \mathrm{PI}^{-}$(early apoptotic events) and Annexin $\mathrm{V}^{+} / \mathrm{PI}^{+}$(late apoptotic/necrotic events); results 
Table I. Effect of VPA on Ara-C sensitivity in patients with AML.

\begin{tabular}{|c|c|c|c|c|c|c|c|c|c|}
\hline \multirow[b]{2}{*}{ No. } & \multirow[b]{2}{*}{ Age } & \multirow[b]{2}{*}{ Gender } & \multirow[b]{2}{*}{ Cytogenetics } & \multirow[b]{2}{*}{$\begin{array}{l}\mathrm{VPA} \mathrm{IC}_{50} \\
(\mathrm{nmol} / \mathrm{l})\end{array}$} & \multicolumn{3}{|c|}{ Ara-C IC ${ }_{50}(\mathrm{nmol} / \mathrm{l})$} & \multicolumn{2}{|c|}{ CI } \\
\hline & & & & & $\begin{array}{c}0 \mathrm{mmol} / \mathrm{l} \\
\mathrm{VPA}\end{array}$ & $\begin{array}{c}0.5 \mathrm{mmol} / \mathrm{l} \\
\mathrm{VPA}\end{array}$ & $\begin{array}{l}1 \mathrm{mmol} / \mathrm{l} \\
\mathrm{VPA}\end{array}$ & $\begin{array}{c}0.5 \mathrm{mmol} / 1 \\
\mathrm{VPA}\end{array}$ & $\begin{array}{c}1 \mathrm{mmol} / 1 \\
\mathrm{VPA}\end{array}$ \\
\hline AML\#1 & 46 & $\mathrm{~F}$ & inv(16) & 3.04 & 4,956 & 3,464 & 2,318 & 0.86 & 0.80 \\
\hline AML\#2 & 26 & M & $\mathrm{t}(8 ; 21)$ & 0.78 & 1,024 & 258 & ND & 0.89 & NA \\
\hline AML\#3 & 28 & $\mathrm{~F}$ & normal & 1.63 & 2,822 & 1,622 & 572 & 0.88 & 0.82 \\
\hline AML\#4 & 40 & M & complex & 5.38 & 7,062 & 4,270 & 2,445 & 0.69 & 0.53 \\
\hline AML\#5 & 53 & M & $\mathrm{t}(6 ; 9)$ & 2.97 & 3,984 & 2,075 & 1,252 & 0.69 & 0.65 \\
\hline AML\#6 & 47 & M & not available & 2.70 & 8,440 & 5,780 & 3,473 & 0.87 & 0.78 \\
\hline AML\#7 & 59 & $\mathrm{~F}$ & normal & 0.91 & 804 & 145 & ND & 0.73 & NA \\
\hline
\end{tabular}

VPA, valproic acid; Ara-C, cytarabine; AML, acute myeloid leukemia; F, female; M, male; ND: no data, NA: not available.

Table II. Primer sequences for RT-qPCR.

\begin{tabular}{lll}
\hline Gene & \multicolumn{1}{c}{ Forward primer } & \multicolumn{1}{c}{ Reverse primer } \\
\hline p53 & ATGGAGGAGCCGCAGTCAGATCCTAC & TCAGTCTGAGTCAGGCCCTTGTGTC \\
Bcl-2 & TGGGATGCCTTTGTGGAACT & ACAGCCAGGAGAAATCAAACAG \\
BAX & TGGAGCTGCAGAGGATGATTG & AGCTGCCACCCGGAAGA \\
FAS & CTCCAAGGGATTGGAATT GA & TTGGTGTTGCTGGTGAGTGT \\
CASP3 & TGGAATTGATGCGTGATGTT & GTCGGCATACTGTTTCAGCA \\
BIRC5 & CAGAGGAGGCGCCAAGACAG & CCTGACGGCGGAAAACGC \\
E2F1 & ATGTTTTCCTGTGCCCTGAG & ATCTGTGGTGAGGGATGAGG \\
BAD & CCTCAGGCCTATGCAAAAAG & AAACCCAAAACTTCCGATGG \\
Bcl-xL & GGTGAGTCGGATTGCAAG & GGCAGTTAGGGATCTCCA \\
CASP9 & ATGGAGGAGGCTGACCGGCAACTCCTG & TCATGAAGTTTTAAAGAACAGCTTCTTC \\
GAPDH & TGTGTCCGTCGTGGATCTGA & TTGCTGTTGAAGTCGCAGGAG \\
ACTB & ATGGAGGGGAATACAGCCC & TTCTTTGCAGCTCCTTCGTT
\end{tabular}

ACTB, $\beta$-actin; BIRC5, survivin; CASP3, caspase-3; CASP9, caspase-9; Bcl, B-cell lymphoma; E2F1, E2F transcription factor 1.

are expressed as the percentage of Annexin $\mathrm{V}^{+}$cells after subtracting the results for untreated cells.

Cell cycle analysis. In the present study, for the analysis of DNA content only, the harvested cells were fixed in $70 \%$ ethanol and stained with $50 \mu \mathrm{g} / \mathrm{ml}$ PI. The DNA content was analyzed by flow cytometric analysis (FACScan). For the determination of DNA and RNA content, in order to distinguish G0 from G1 cells, the harvested cells were fixed and permeabilized using a BD IntraSure kit, and subsequently intracellularly stained with an antibody against Ki-67 and with Hoechst 33342 (both from BD Bioscience). Data analysis was performed using FlowJo version 7.0 (TreeStar, Ashland, OR, USA).

Reverse transcription-quantitative polymerase chain reaction ( $R T-q P C R)$. Total RNA was extracted using TRIzol reagent (Invitrogen) and first-strand cDNA was synthesized using ReverTra Ace qPCR RT Master Mix (Toyobo, Osaka, Japan). RT-qPCR was performed in an ABI PRISM 7500 Fast Real-
Time PCR system using SYBR ${ }^{\circledR}$ Premix Ex Taq ${ }^{\mathrm{TM}}$ (Tli RNase H Plus) according to the manufacturer's protocol (Takara, Otsu, Japan). Table II lists the primer sequences used.

Western blot analysis. Leukemic cells were lysed in $20 \mathrm{mM}$ Tris buffer, $\mathrm{pH} 7.4$, containing $0.14 \mathrm{M} \mathrm{NaCl}, 1 \% \mathrm{NP}-40,10 \%$ glycerol, $1 \mathrm{mM}$ sodium orthovanadate and protease inhibitors (Roche, Basel, Switzerland). Protein concentration levels were determined using the Bradford assay (Bio-Rad, Hercules, CA, USA). Total protein $(50 \mu \mathrm{g})$ was resolved on a $10 \%$ SDS-PAGE gel, transferred to a nitrocellulose membrane, blocked for $1 \mathrm{~h}$ with a $50 \mathrm{mM}$ Tris buffer, $\mathrm{pH} 7.5$, containing $0.15 \mathrm{M} \mathrm{NaCl}$, $0.05 \%$ Tween-20 (TBST), and 5\% (wt/vol) skimmed milk, and probed overnight at $4^{\circ} \mathrm{C}$ with primary antibody for acetylhistone 3, acetyl-histone 4, B-cell lymphoma (Bcl)-2, BAD, BAX and GAPDH (Cell Signaling Technology, Danvers, MA, USA). The blot was washed with TBST buffer, incubated with the corresponding horseradish peroxidase-conjugated secondary antibody for $1 \mathrm{~h}$, and visualized by enhanced chemiluminescence (Pierce, Rockford, IL, USA). 
A

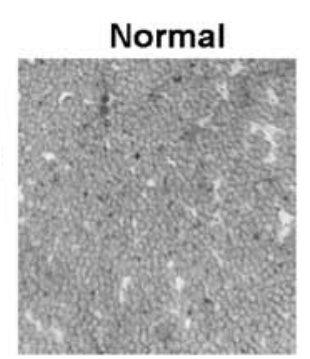

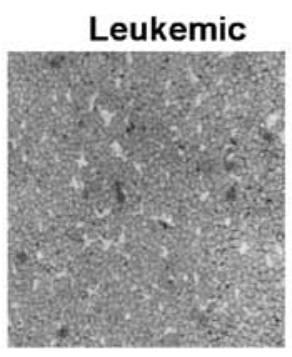

B

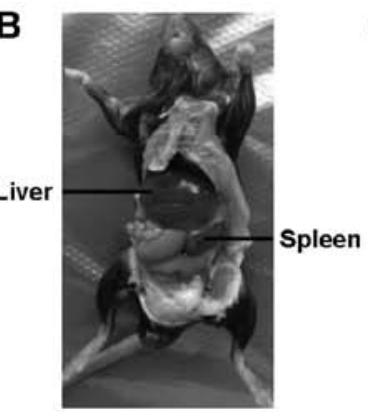

C

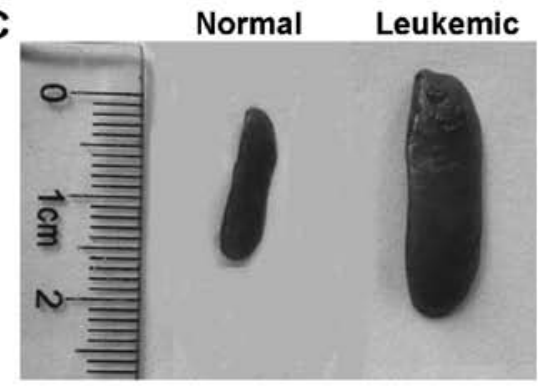

D

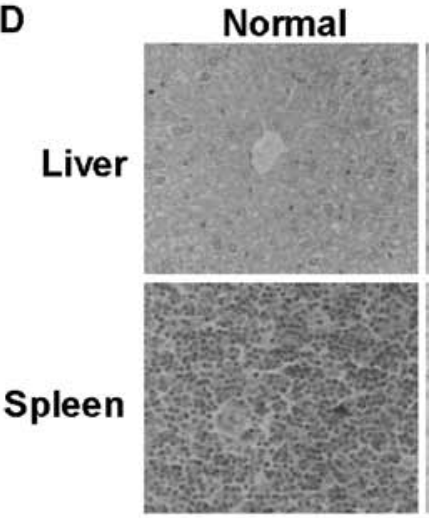

Leukemic
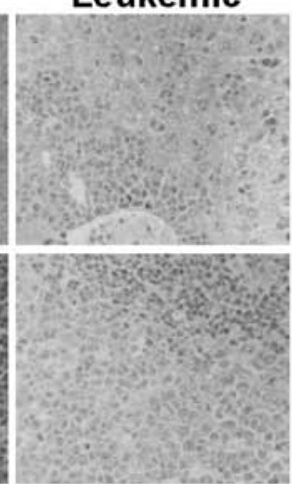

E
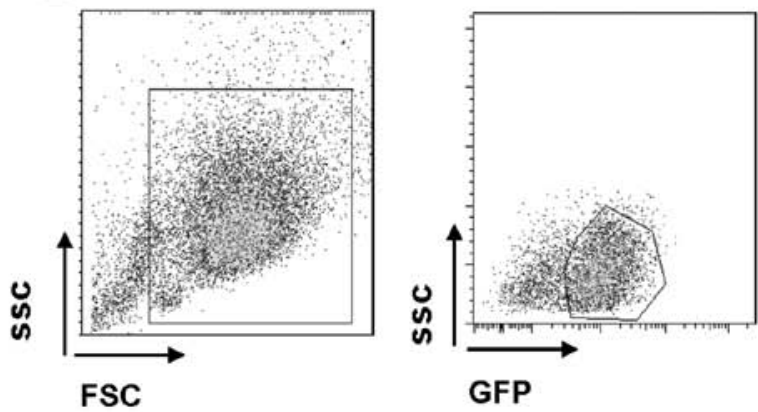

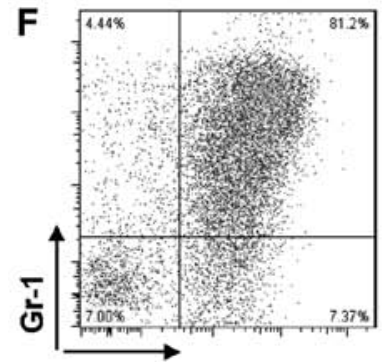

Mac-1
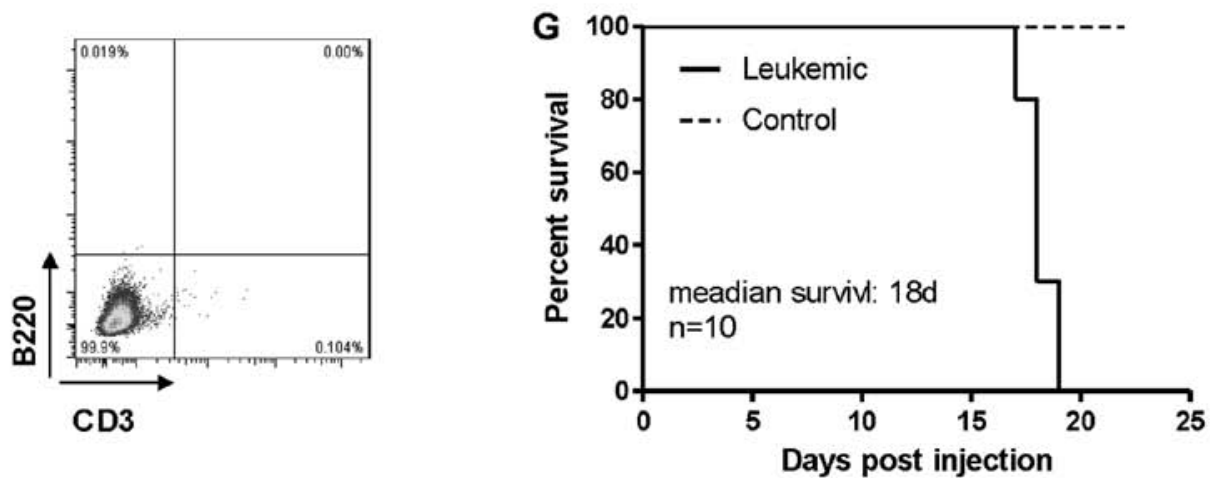

Figure 1. Construction of non-irradiated mouse model of acute myeloid leukemia (AML). (A) Peripheral blood (PB) smears of normal and leukemic mice. (B) Anatomical distribution of organ involvement in mice with AML. (C) Comparative spleen size in mice with AML vs. normal mice. (D) Histological evaluation of a spleen and liver from representative normal and leukemic mice (magnification, $x 40)$. (E) Mice were injected with leukemia cells, sacrificed when moribund, and $\mathrm{GFP}^{+}$leukemia cells in the bone marrow were assessed by flow cytometry. (F) Flow cytometric analysis of the leukemia cell phenotype. (G) The $100 \%$ penetrance of the leukemic development is shown in the survival curve, with a median latency of 18 days ( $\mathrm{n}=10$ ).

BAX gene silencing with siRNAs. Gene silencing was achievedwith siRNAs (sense and antisense strands; sequence: 5'-CTCC GGCGAATTGGAGATGAA-3'), which were purchased from Guangzhou RiboBio (Guangzhou, China). Cells were transfected with siRNAs using Lipofectamine 2000 (Invitrogen) according to the manufacturer's instructions for $48 \mathrm{~h}$ unless otherwise stated. Following transfection, cells were harvested for experiments as described for parental THP-1 cells.

Statistical analysis. Data are expressed as the standard error of the means \pm standard deviation (SD) of three or more separate experiments performed in duplicate. ANOVA was used for significance testing, and a P-value $<0.05$ was considered to indicate a statistically significant difference. Statistical analyses were performed with GraphPad Prism 5.0 software (GraphPad Software, La Jolla, CA, USA).

\section{Results}

Construction of non-irradiated mouse model of AML. In order to gain insight into the effects of VPA and Ara-C on leukemic cells in vivo, we established a non-irradiated mouse model of acute myeloid leukemia, as previously described (18). Briefly, Lin ${ }^{-}$cells $\left(\mathrm{CD} 45.2^{+}\right)$were isolated from the $\mathrm{BM}$ of $\mathrm{C} 57 \mathrm{BL} / 6 \mathrm{~J}$ mice and transduced with the MSCV-MLL-AF9-IRES-GFP vector. A total of $1 \times 10^{6}$ transduced cells $\left(\mathrm{GFP}^{+}\right)$were transplanted into lethally irradiated recipients (9.5 Gy, C57BL/6J). Leukemic cells $\left(1 \times 10^{6}\right)$ which were harvested from diseased mice that had clinically evident leukemia 4 weeks after transplantation were injected into non-irradiated recipients (B6.SJL, CD45.1 ${ }^{+}$). With this robust leukemia model, $100 \%$ of the mice that received leukemic cells developed AML (Fig. 1G). 

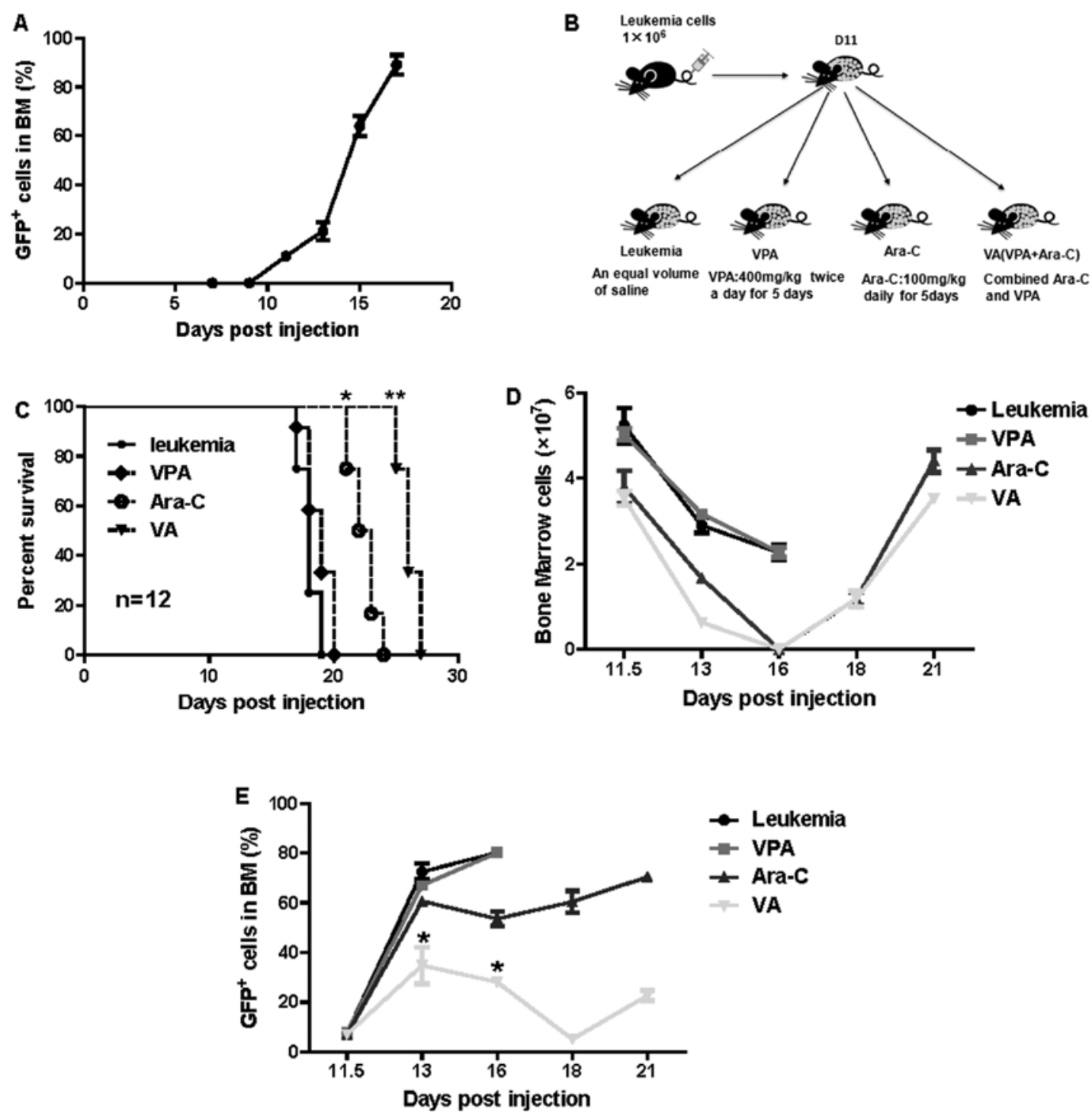

Figure 2. Survival of leukemic mice improves after combined valproic acid (VPA) and cytarabine (Ara-C) treatment. (A) The percentage of GFP leukemic cells in bone marrow (BM) markedly increased. (B) Schematic presentation of leukemic mice grouping and treatment. (C) Kaplan-Meier survival curves of leukemic mice that received saline, VPA, Ara-C or combined VPA and Ara-C $\left(\mathrm{n}=12\right.$ per group; ${ }^{\mathrm{P}}<0.05$ Ara-C group vs. control group; ${ }^{* *} \mathrm{P}<0.05 \mathrm{VA}$ group vs. control group). (D) Number of bone marrow mononuclear cells (BMNCs) in untreated, VPA-treated, Ara-C-treated, and combined drug-treated leukemic mice on days 11.5, 13, 16, 18 and 21 after modeling. (E) Percentage of leukemia cells in the BM of the four groups ("P<0.05 VA group vs. Ara-C group). In (A, D and $\mathrm{E})$ for each time point, $\mathrm{n}=3-5$ per group.

Mice with acute myeloid leukemia induced by the $M L L-A F 9$ oncogene demonstrated progressive anemia and leukocytosis (Fig. 1A), with extensive hepatosplenomegaly (Fig. 1B and C). Upon reaching a terminal stage, GFP-positive cells dominated the peripheral blood, bone marrow, spleen and liver (Fig. 1D and E). Immunophenotyping revealed that $M L L-A F 9$ leukemia predominantly consisted of differentiated myeloid cells $\left(\mathrm{Macl}^{+}, \mathrm{Gr}^{+}, \mathrm{CD}^{-}, \mathrm{B} 220^{-}\right.$and $\mathrm{GFP}^{+}$) and involved the monocytic lineage, which is a typical feature of human 11q23-rearranged AML (Fig. 1E and F).

Combined VPA and Ara-C treatment prolongs the survival of leukemic mice. At +11 days after transplantation, when the leukemic burden reached $10 \pm 2 \%$ of mononuclear cells in bone marrow, mice received chemotherapy (Fig. 2A and B). Leukemic mice were randomized into four groups and treated for 5 consecutive days by intraperitoneal injec- tion with one of four treatments. The first group (control) received PBS only, the second received VPA $(400 \mathrm{mg} / \mathrm{kg}$, every $12 \mathrm{~h})$ and the third received Ara-C (100 mg/kg, every $24 \mathrm{~h}$ ). The fourth group received VPA (400 mg/ $\mathrm{kg}$, every $12 \mathrm{~h}$ ) and Ara-C (100 mg/kg, every $24 \mathrm{~h})$. Survival analysis was measured from the day of leukemia cell injection. The median survival time of the mice in the control group was 18 days, while treatment with VPA alone did not markedly prolong the survival of the leukemic mice (19 vs. 18 days, $\mathrm{P}>0.05$ ). Compared with the control group, the third group (Ara-C) mice lived much longer (median survival: 22 vs. 18 days, $\mathrm{P}<0.0001$ ), whereas for the mice treated with VPA and Ara-C, survival was significantly increased to 26 days. The administration of the combination of VPA and Ara-C resulted in a statistically significant increase in the overall survival of leukemic mice when compared with AraC-treated mice (Fig. 2C; $\mathrm{P}=0.0172$ ). 
Table III. $\mathrm{IC}_{50}$ values of VPA and Ara-C alone according to duration of treatment.

\begin{tabular}{lccccccc}
\hline & \multicolumn{3}{c}{ VPA $(\mathrm{mmol} / \mathrm{l})$} & & \multicolumn{3}{c}{ Ara-C $(\mu \mathrm{mol} / \mathrm{l})$} \\
\cline { 2 - 4 } \cline { 7 - 8 } Cell line & $24 \mathrm{~h}$ & $48 \mathrm{~h}$ & $72 \mathrm{~h}$ & & $24 \mathrm{~h}$ & $48 \mathrm{~h}$ & $72 \mathrm{~h}$ \\
\hline THP-1 & $6.72 \pm 0.32$ & $1.47 \pm 0.10$ & $1.77 \pm 0.11$ & & $3.03 \pm 0.16$ & $3.44 \pm 0.18$ & $3.14 \pm 0.15$ \\
K562 & - & $4.23 \pm 0.23$ & $2.46 \pm 0.12$ & & - & $2.59 \pm 0.16$ & $2.70 \pm 0.10$ \\
HL-60 & - & - & $5.15 \pm 0.35$ & & - & - & $3.27 \pm 0.27$ \\
\hline
\end{tabular}

Viable cells were measured using the CCK- 8 assay. $\mathrm{IC}_{50}$ values are presented as the means \pm standard errors of at least three independent experiments. -, could not be calculated. VPA, valproic acid; Ara-C, cytarabine.

Table IV. Effect of VPA on Ara-C sensitivity in the AML cell lines.

\begin{tabular}{|c|c|c|c|c|c|c|}
\hline \multirow[b]{2}{*}{ Cell line } & \multirow[b]{2}{*}{$\begin{array}{l}\mathrm{VPAIC}_{50} \\
(\mathrm{mmol} / \mathrm{l})\end{array}$} & \multicolumn{3}{|c|}{ Ara-C IC I0 $_{50 \mathrm{~mol} / \mathrm{l})}$} & \multicolumn{2}{|c|}{$\mathrm{CI}$} \\
\hline & & $\begin{array}{c}0 \mathrm{mmol} / \mathrm{l} \\
\mathrm{VPA}\end{array}$ & $\begin{array}{c}0.5 \mathrm{mmol} / 1 \\
\mathrm{VPA}\end{array}$ & $\begin{array}{c}1.0 \mathrm{mmol} / 1 \\
\mathrm{VPA}\end{array}$ & $\begin{array}{c}0.5 \mathrm{mmol} / 1 \\
\mathrm{VPA}\end{array}$ & $\begin{array}{c}1.0 \mathrm{mmol} / \mathrm{l} \\
\mathrm{VPA}\end{array}$ \\
\hline THP-1 & $1.47 \pm 0.10$ & $3.03 \pm 0.16$ & $1.60 \pm 0.05$ & $0.70 \pm 0.01$ & 0.86 & 0.91 \\
\hline K562 & $2.46 \pm 0.12$ & $2.59 \pm 0.16$ & $1.59 \pm 0.07$ & $1.02 \pm 0.02$ & 0.82 & 0.79 \\
\hline HL-60 & $5.15 \pm 0.35$ & $3.27 \pm 0.27$ & $2.32 \pm 0.11$ & $1.57 \pm 0.13$ & 0.80 & 0.67 \\
\hline
\end{tabular}

VPA, valproic acid; Ara-C, cytarabine; AML, acute myeloid leukemia; CI, combination index.

To further examine the effect of Ara-C and/or VPA on the mouse model of leukemia, we examined quantitative changes of bone marrow mononuclear cells and white blood cells, and analyzed the percentage of leukemic cells in both peripheral blood (PB) and BM by FACS. In the untreated leukemic mice, the number of $\mathrm{BM}$ mononuclear cells (NCs) progressively decreased during the development of leukemia, while the percentage of leukemia cells $\left(\mathrm{GFP}^{+}\right)$in the BM was markedly increased. In the group treated with VPA alone, we observed a similar trend in terms of changes for BMNCs and leukemia cells. BMNCs in the group treated with Ara-C alone and the combined treatement group exhibited the same trend: they decreased after therapy and then increased again after the fifth day post-therapy. However, mice treated with combination therapy demonstrated a substantial delay in leukemia cell growth when compared with the mice treated with Ara-C alone (Fig. 2E).

Synergistic antileukemic effect of VPA and Ara-C in the AML cell lines. As explained above, we hypothesized that VPA synergized with Ara-C, resulting in enhanced antileukemic effects in a murine model infected with $M L L-A F 9$ leukemia. To further investigate the mechanism involved, in the present study we examined the impact of VPA on Ara-C cytotoxicities in THP- 1 cells using a CCK- 8 assay. With the increase in concentration of Ara-C or VPA, the growth of human THP-1 cells was significantly suppressed (Fig. $3 \mathrm{~A}$ and $\mathrm{B}$ ). The in vitro cytotoxic activities of VPA and Ara-C are shown in Table III, and $\mathrm{IC}_{50}$ values were determined from these dose response curves. When simultaneously administered with Ara-C, VPA at 0.5 and $1 \mathrm{mmol} / 1$ significantly increased the sensitivity of THP-1 cells to Ara-C by decreasing the $\mathrm{IC}_{50}$ of Ara-C by 1.9- and 4.3-fold, respectively (Fig. 3B and Table IV). To determine whether Ara-C and VPA interacted synergistically, isobologram analysis was performed. This analysis provides a $\mathrm{CI}$ value that measures the degree of interaction between two or more drugs, where a $\mathrm{CI}<1$ and a $\mathrm{CI}>1$ indicate synergism and antagonism, respectively. As shown in Table IV and Fig. 3C, CI values $<1$ were calculated from these data, indicative of synergism. Analogous results were obtained when K562 and HL-60 cells were evaluated following cotreatment with Ara-C and VPA (Table IV).

Furthermore, to determine whether the synergistic interactions also occurred in primary, genetically defined AML specimens, 7 primary AML cultures were established in vitro and treated with Ara-C and/or VPA (Table I). When administered as a single agent, Ara-C inhibited proliferation and reduced cell viability in a dose-dependent manner in 7 primary AML cultures with $\mathrm{IC}_{50}$ ranging from $804-8,840 \mathrm{nmol} / \mathrm{l}$. We then explored the effect of VPA ( 0.5 and $1 \mathrm{mmol} / \mathrm{l})$ combined with the sample-specific $\mathrm{IC}_{50}$ dose of Ara-C on 7 AML primary cultures. We demonstrated that simultaneous administration of VPA and induced a greater decrease in cell viability than Ara-C alone (Table I). Collectively, our results showed that the synergistic antileukemic effect of combined VPA and Ara-C was universal in AML subtypes.

VPA and Ara-C synergistically induces apoptosis in AML cells. VPA and Ara-C exerted synergistic antileukemic effects in the myeloid leukemiacell lines. We hypothesized that Ara-C-induced apoptosis was enhanced by the addition of VPA. To test the concept, we treated THP-1 and K562 cells with Ara-C and/or VPA, and the percentage of apoptotic cells was determined by Annexin V/PI staining followed by flow cytometric analysis. We noted that combined VPA and Ara-C-treated cultures contained 

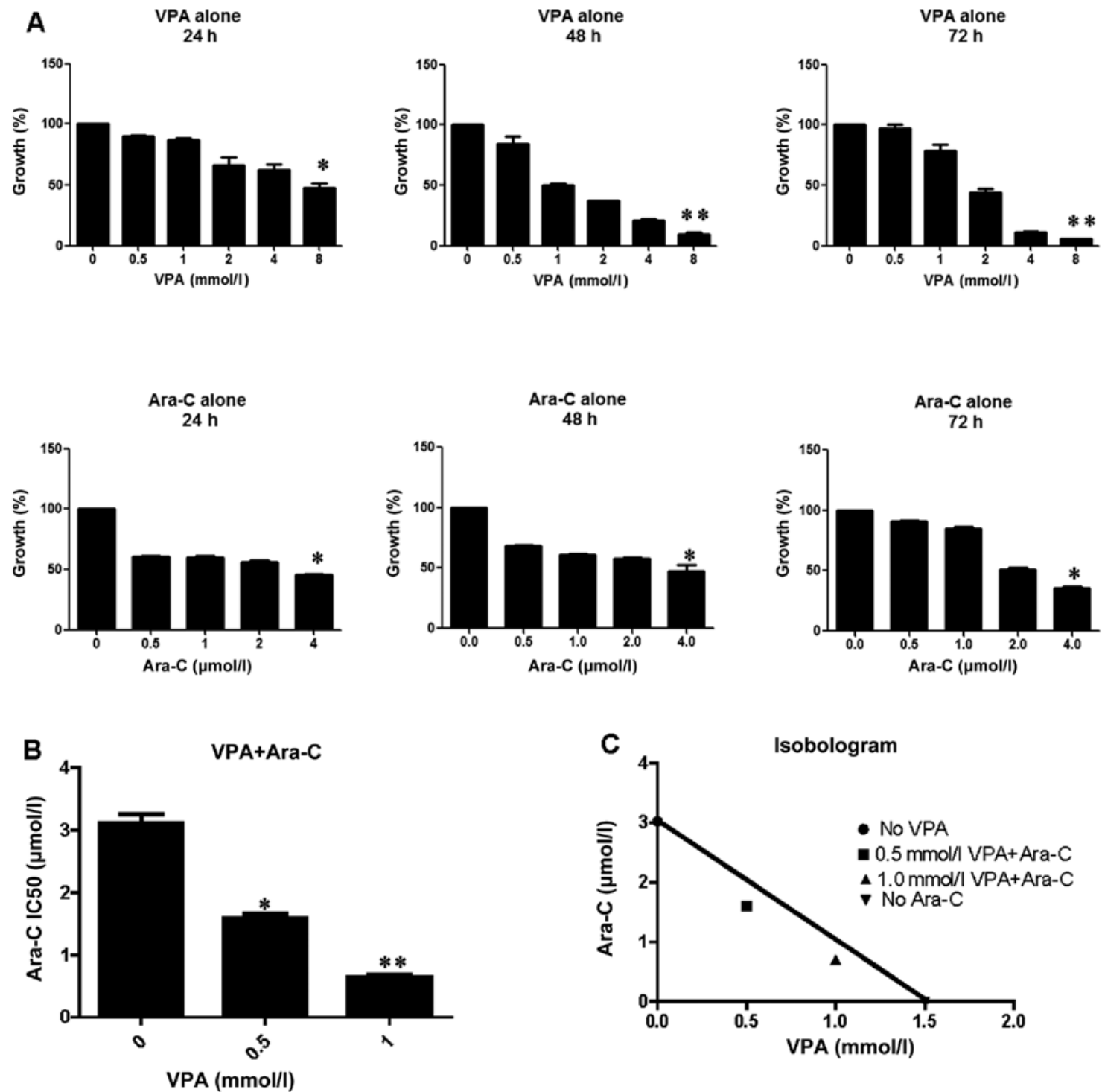

Figure 3. Synergistic antileukemic effect of valproic acid (VPA) and cytarabine (Ara-C) on acute myeloid leukemia (AML) cell lines. (A) THP-1 cells treated with the indicated concentrations of Ara-C or VPA for $72 \mathrm{~h}$, and cell viability was analyzed by CKK-8 assay. (B) $\mathrm{IC}_{50}$ of Ara-C when THP-1 cells treated with Ara-C alone or combined with VPA. (C) Standard isobologram for analyzing the antileukemic interaction between VPA and Ara-C in THP-1 cells. Points on, below and above the curve denote additive, synergistic and antagonistic effects of the drug combinations, respetively. ${ }^{*} \mathrm{P}<0.05$ and ${ }^{* *} \mathrm{P}<0.001$ treatment group vs. control group.

predominantly more apoptotic cells, compared with the results of individual drug treatments (Fig. 4A and B).

To determine whether cell-cycle arrest contributed to growth inhibition, cellular DNA content distribution was examined by flow cytometry (Fig. 4E). Treatment with Ara-C alone resulted in $\mathrm{S}$ phase blockade compared with untreated cells (Fig. 4F). We also used a combination of intracellular Ki-67 and Hoechst 33342 labeling to study the cell cycle distributions (Fig. 4C). When treated with VPA alone, the percentage of cells in the G2/M phase gradually decreased, and the vast majority of cells appeared to arrest at G0/G1 phase (Fig. 4D). In both cell lines, co-treatment with VPA and Ara-C resulted in the accumulation of cells in the G1 phase.

$B A X$ plays a critical role in apoptosis induced by combined treatment with VPA and Ara-C. To explore the molecular mechanism underlying Ara-C and/or VPA-induced cell proliferation arrest and apoptosis, we examined the expression of 10 apoptosis-related genes in THP-1 and K562 cells. Results from RT-qPCR showed that Ara-C and VPA significantly upregulated mRNA levels of $B A X$ in both THP-1 and K562 cells (Fig. 5A and B). Moreover, mRNA levels of $B A X$ were significantly increased by treatment with a combination of Ara-C and VPA when compared to treatment with Ara-C alone. The synergistic effect of VPA and Ara-C on $B A X$ expression was also confirmed by western blot analysis (Fig. 5C).

To provide direct evidence that $B A X$ is a critical effector of the antileukemic effects of combined VPA and Ara-C treatment, we designed a siRNA against $B A X$ to knockdown $B A X$ expression in THP-1 cells. $B A X$ siRNA was used to transfect THP-1 cells, and RT-qPCR was performed to measure $B A X$ expression. As shown in Fig. 5D, transfection with $B A X$ siRNA markedly decreased $B A X$ expression in a timedependent manner. The effects of $B A X$ knockdown on THP-1 


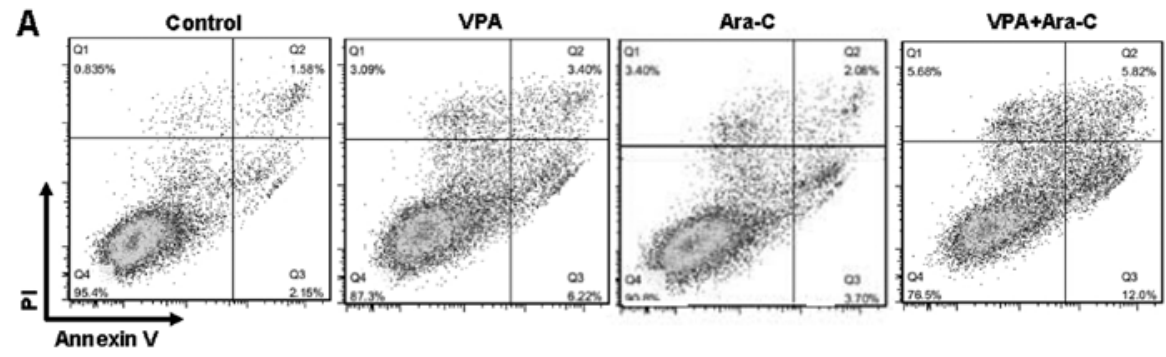

B
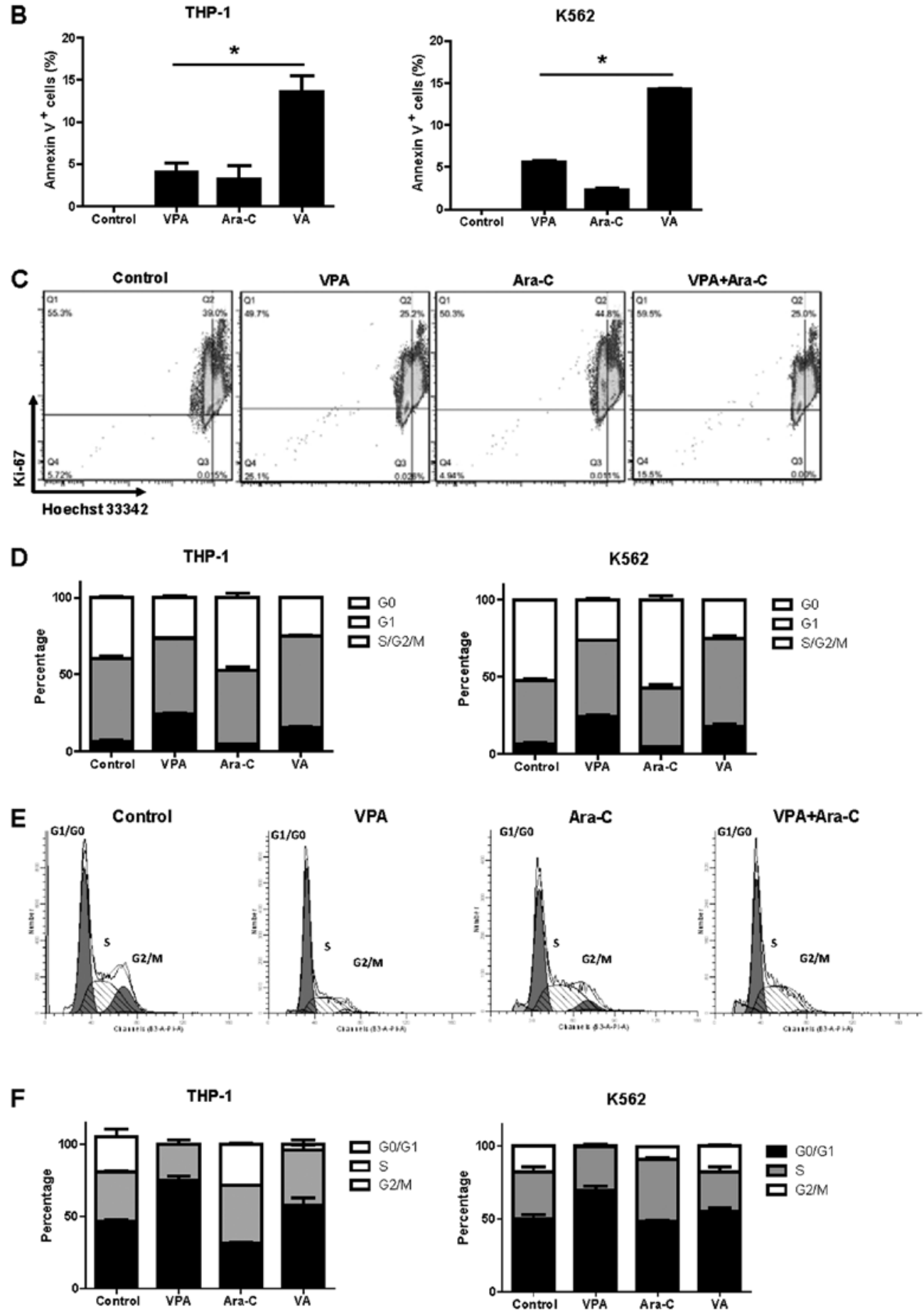

Figure 4. Valproic acid (VPA) and cytarabine (Ara-C) synergistically induce acute myeloid leukemia (AML) cell apoptosis. (A) Flow cytometric analysis of THP-1 cells after treatment with combined VPA and Ara-C or individual treatme. Annexin $\mathrm{V}^{+} / \mathrm{PI}^{-}$indicates early apoptotic events; Annexin $\mathrm{V}^{+} / \mathrm{PI}^{+}$indicates late apoptotic/necrotic events. (B) Apoptosis analysis of THP-1 and K562 cells after treatment, expressed as the percentage of Annexin $\mathrm{V}^{+}$cells after subtracting the results for the untreated cells. (C) Cell cycle flow charts of THP-1 cells stained with Ki67/Hoechst 33342. Ki67-Hoechst', Ki67+Hoechst and Ki67 ${ }^{+}$Hoechst ${ }^{+}$indicate G0, G1 and S/G2/M phases, respectively. (D) THP-1 and K562 cells treated with combined Ara-C or VPA or individual treatment. Cell cycle was analyzed by staining with Ki67/Hoechst 33342. (E) Cell cycle of PI-stained THP-1 cells. (F) THP-1 and K562 cells treated with Ara-C or VPA combined or individually. Cell cycle distribution was analyzed by staining with PI. " $\mathrm{P}<0.05$ VA group vs. Ara-C group. 
A

THP-1

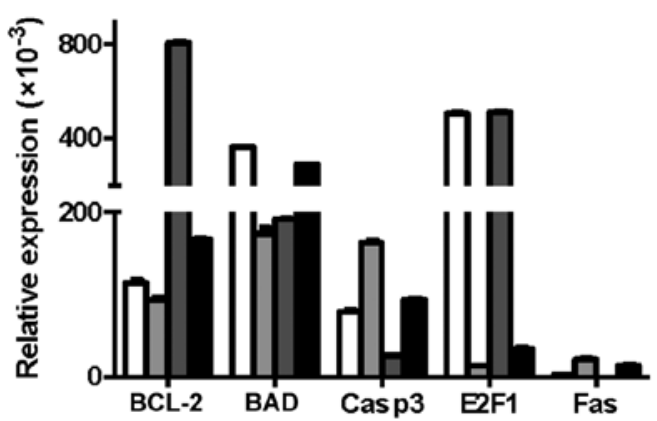

B

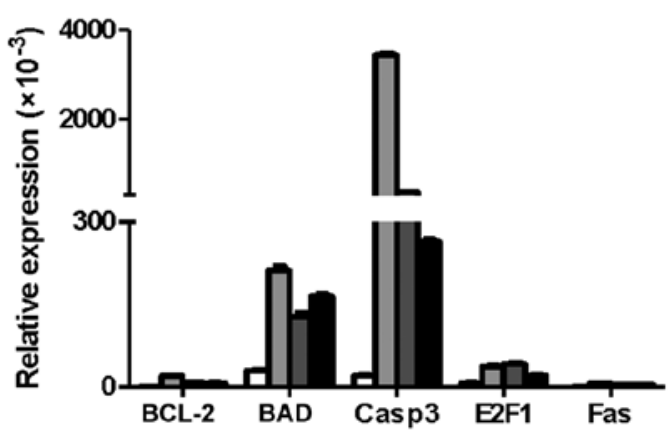

C

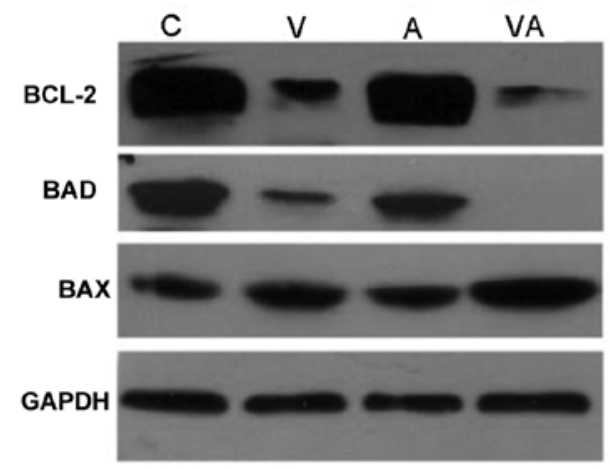

THP-1
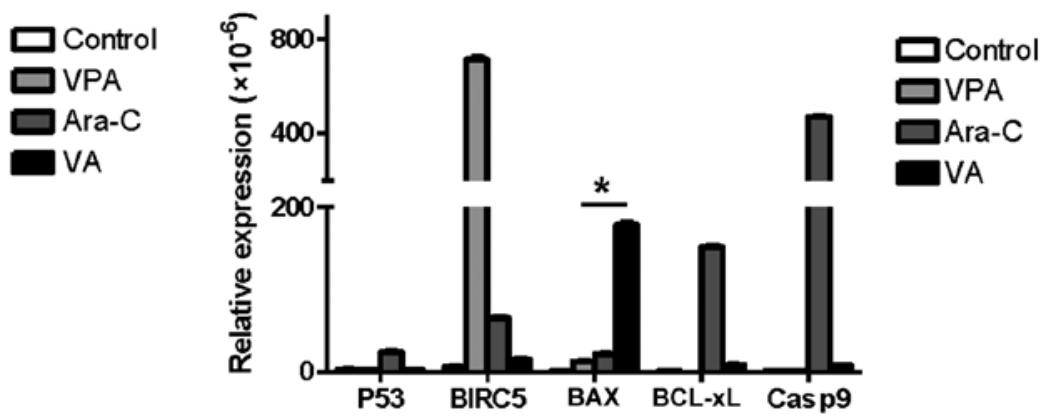

K562

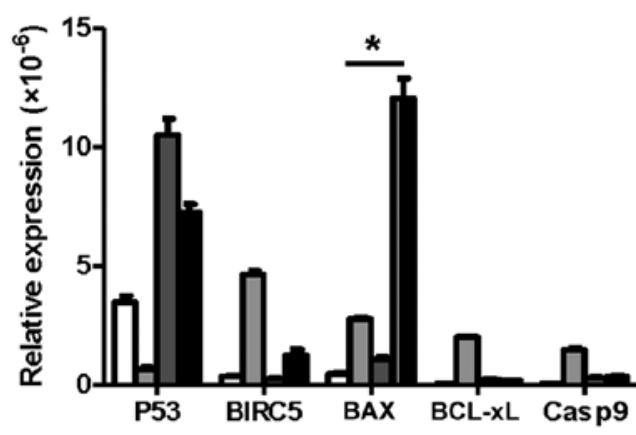

D

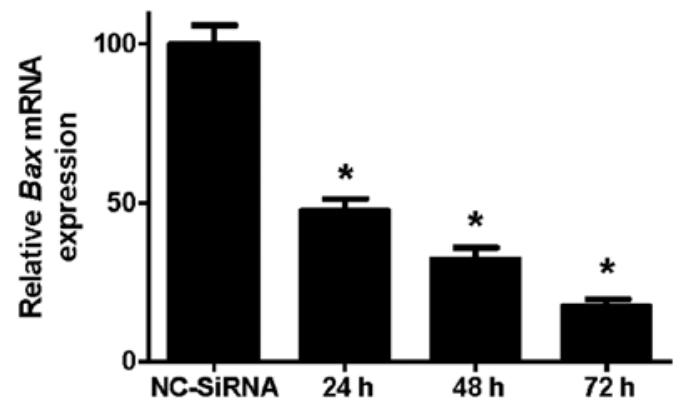

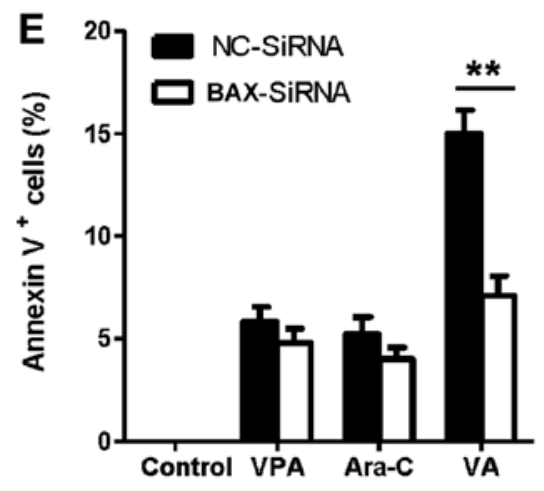

Figure 5. BAX plays a critical role in apoptosis induced by combined valproic acid (VPA) and cytarabine (Ara-C). (A) RT-qPCR of mRNA levels of apoptosisrelated genes in THP-1 cells after treatment with combined VPA and Ara-C or individual treatment. Histograms show relative mRNA levels normalized to the control GAPDH gene. Relative expression $=2^{- \text {(CTx-CTr) }}$, where $\mathrm{CTx}$ is the threshold cycle $(\mathrm{CT})$ value of the target gene and $\mathrm{CTr}$ is the $\mathrm{CT}$ value of the reference gene. (B) RT-qPCR of mRNA levels of apoptosis-related genes in K562 cells. (C) Western blot analysis of B-cell lymphoma (BCL)-2, BAD, BAX and GAPDH in THP-1 cells. C, control; A, Ara-C; V, VPA; VA, VPA + Ara-C. (D) Downregulation of BAX mRNA expression by siRNA in THP-1 cells. Cells were transfected with negative control (NC) siRNA or BAX siRNA for 24, 48 and $72 \mathrm{~h}$. Relative mRNA expression was measured using RT-qPCR. The results are expressed as the means $\pm \mathrm{SD}(\mathrm{n}=3)$. ${ }^{*} \mathrm{P}<0.05$ vs. NC siRNA. (E) THP-1 cells transfected with $B A X$ siRNA or NC siRNA. Flow cytometric analysis of apoptosis after treatment with combined VPA and Ara-C or individual treatment. ${ }^{*} \mathrm{P}<0.05$ and ${ }^{* * *} \mathrm{P}<0.005$. 


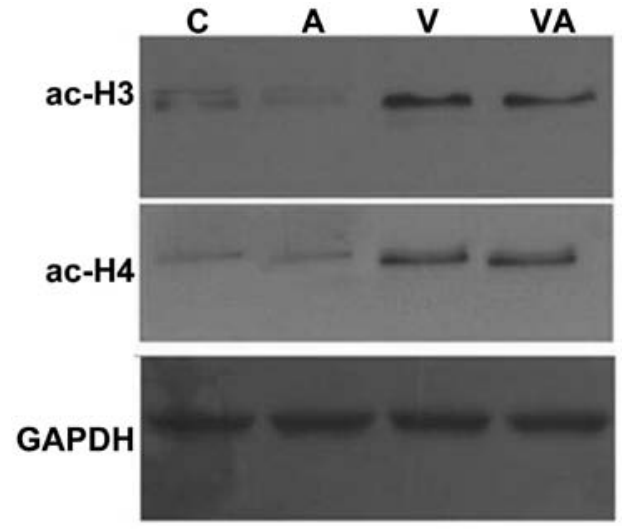

Figure 6. Valproic acid (VPA) treatment increases the levels of histone H3 and $\mathrm{H} 4$ acetylation. Western blot analysis of acetylated $\mathrm{H} 3, \mathrm{H} 4$ and GAPDH in THP-1 cells. C, control; A, cytarabine; V, VPA; VA, VPA + Ara-C.

cell apoptosis was analyzed by flow cytometric analysis. After $48 \mathrm{~h}$ of treatment, the percentage of apoptotic cells decreased to $7.1 \%$, as compared to negative control-transfected cells (15.0\%) (Fig. 5E, $\mathrm{P}<0.005)$.

VPA treatment increases the level of histone $\mathrm{H} 3$ and $\mathrm{H} 4$ acetylation. In this study, histone $\mathrm{H} 3$ and $\mathrm{H} 4$ acetylation was evaluated by western blot analysis, and total GAPDH was the control (Fig. 6). Treatment with VPA alone and combined VPA/Ara-C treatment resulted in a more noticeable increase in histone $\mathrm{H} 3$ and $\mathrm{H} 4$ acetylation in THP-1 cells, compared to treatment with Ara-C alone. Taken together, the results of our experiments suggest that VPA-induced histone acetylation synergistically enhanced cytarabine sensitivities in myeloid leukemia cell lines, diagnostic blasts and the murine model of leukemia.

\section{Discussion}

VPA has been used as an antiepileptic drug for decades, though it has also been demonstrated that VPA acts as a histone deacetylase inhibitor, which has an additive effect on growth inhibition, the induction of apoptosis and cell cycle arrest $(10,19,20)$. However, according to the results from clinical studies on the effects of VPA on AML, it has been noted that VPA exerts beneficial effects but does not exert remarkable clinical effects when used as a single-agent therapy $(21,22)$. In this context, we hypothesized that combining VPA with other antileukemic agents would be a useful and promising strategy to improve the antileukemic efficiency of VPA. Our previous study showed that combining VPA with curcumin, a well-known dietary pigment derived from Curcuma longa, exerted substantial and synergistic anticancer effects, which led to the proliferation arrest, death and G1 phase-accumulation of HL-60 cells (23). In addition, we also demonstrated that the combination of VPA and curcumin led to the upregulated expression of genes associated with apoptosis, which was accompanied by increased histone acetylation in the promoter region.

In the present study, we established a non-irradiated murine model with $M L L-A F 9$ leukemia, that accurately reflected the genetics and pathology of human AML, as a reliable preclinical system for studying the therapeutic response of a given drug and also a combination of drugs. In mice treated with
VPA and Ara-C, we noted a marked delay in the progression of leukemia, which led to prolongation of the survival of mice in the murine leukemia model. To the best of our knowledge, this is the first time that experiments have demonstrated the effect of combination Ara-C and VPA treatment on leukemic mice in vivo. Analogous results were obtaine $5 \mathrm{~d}$ when myeloid leukemia cell lines and diagnostic blast samples were evaluated after treatment with Ara-C and VPA in vitro.

Furthermore, we demonstrated that a synergistic antileukemic effect occurred between Ara-C and VPA in cell lines and primary AML samples in vitro. Our results showed that the combination regimen led to the synergistic induction of mitochondrial damage and apoptosis in human leukemia cells. In addition, we revealed that co-exposure of cells to VPA and Ara-C arrested the cell cycle in the G0/G1 phase in both $\mathrm{K} 562$ and THP-1 cells. The combination regimen also induced the expression of $B A X$, an apoptosis-related protein, in THP-1 cells. Moreover, it is known that treatment with HDAC inhibitors promotes $B A X$-dependent apoptosis in several cell types $(24,25)$. In the present study, we showed that $B A X$ also plays critical roles in Ara-C and VPA-induced apoptosis in AML cells.

In conclusion, taken together, we demonstrated that VPA in combination with Ara-C and VPA resulted in synergistic anti-leukemic activity in the murine leukemic model, AML cell lines and patient samples. The combination significantly upregulated the expression of $B A X$, and finally led to the proliferation arrest, G0/G1 phase-accumulation and apoptosis of AML cells. These results provide evidence that VPA is an attractive prospect for use in combination therapy with other antileukemic agents.

\section{Acknowledgements}

This study was supported by grants from the National Natural Science Foundation of China (nos. 81090413, 81270638, 81270567, 81470321 and 81300416).

\section{References}

1. Estey EH: Acute myeloid leukemia: 2014 update on risk-stratification and management. Am J Hematol 89: 1063-1081, 2014.

2. Chen X, Xie H, Wood BL, Walter RB, Pagel JM, Becker PS, Sandhu VK, Abkowitz JL, Appelbaum FR and Estey EH: Relation of clinical response and minimal residual disease and their prognostic impact on outcome in acute myeloid leukemia. J Clin Oncol 33: 1258-1264, 2015.

3. Fong CY, Grigoriadis G, Hocking J, Coutsouvelis J, Muirhead J, Campbell P, Paul E, Walker P, Avery S, Patil S, et al: Fludarabine, cytarabine, granulocyte-colony stimulating factor and amsacrine: an effective salvage therapy option for acute myeloid leukemia at first relapse. Leuk Lymphoma 54: 336-341, 2013.

4. Yoon JH, Cho BS, Kim HJ, Kim JH, Shin SH, Yahng SA, Lee SE, Eom KS, Kim YJ, Lee S, et al: Outcomes of elderly de novo acute myeloid leukemia treated by a risk-adapted approach based on age, comorbidity, and performance status. Am J Hematol 88: 1074-1081, 2013.

5. Kim SJ, Cheong JW, Kim DY, Lee JH, Lee KH, Kim YK, Kim HJ, Song IC, Jo DY, Lee JO, et al; Korean Society of Hematology AML/MDS Working Party: role of induction and consolidation chemotherapy in elderly acute myeloid leukemia patients. Int J Hematol 100: 141-151, 2014.

6. Pilatrino C, Cilloni D, Messa E, Morotti A, Giugliano E, Pautasso M, Familiari U, Cappia S, Pelicci PG, Lo Coco F, et al: Increase in platelet count in older, poor-risk patients with acute myeloid leukemia or myelodysplastic syndrome treated with valproic acid and all-trans retinoic acid. Cancer 104: 101-109, 2005. 
7. Quintás-Cardama A, Santos FP and Garcia-Manero G: Histone deacetylase inhibitors for the treatment of myelodysplastic syndrome and acute myeloid leukemia. Leukemia 25: 226-235, 2011.

8. Bots M, Verbrugge I, Martin BP, Salmon JM, Ghisi M, Baker A, Stanley K, Shortt J, Ossenkoppele GJ, Zuber J, et al: Differentiation therapy for the treatment of $t(8 ; 21)$ acute myeloid leukemia using histone deacetylase inhibitors. Blood 123: 1341-1352, 2014.

9. Liss A, Ooi CH, Zjablovskaja P, Benoukraf T, Radomska HS, Ju C, Wu M, Balastik M, Delwel R, Brdicka T, et al: The gene signature in CCAAT-enhancer-binding protein $\alpha$ dysfunctional acute myeloid leukemia predicts responsiveness to histone deacetylase inhibitors. Haematologica 99: 697-705, 2014.

10. Duenas-Gonzalez A, Candelaria M, Perez-Plascencia C, PerezCardenas E, de la Cruz-Hernandez E and Herrera LA: Valproic acid as epigenetic cancer drug: preclinical, clinical and transcriptional effects on solid tumors. Cancer Treat Rev 34: 206-222, 2008.

11. Tang R, Faussat AM, Majdak P, Perrot JY, Chaoui D, Legrand O and Marie JP: Valproic acid inhibits proliferation and induces apoptosis in acute myeloid leukemia cells expressing P-gp and MRP1. Leukemia 18: 1246-1251, 2004

12. Insinga A, Monestiroli S, Ronzoni S, Gelmetti V, Marchesi F, Viale A, Altucci L, Nervi C, Minucci S and Pelicci PG: Inhibitors of histone deacetylases induce tumor-selective apoptosis through activation of the death receptor pathway. Nat Med 11: 71-76, 2005.

13. Cencic R, Carrier M, Trnkus A, Porco JA Jr, Minden M and Pelletier J: Synergistic effect of inhibiting translation initiation in combination with cytotoxic agents in acute myelogenous leukemia cells. Leuk Res 34: 535-541, 2010.

14. Jiang C, Hu X, Wang L, Cheng H, Lin Y, Pang Y, Yuan W, Cheng $\mathrm{T}$ and Wang $\mathrm{J}$ : Excessive proliferation and impaired function of primitive hematopoietic cells in bone marrow due to senescence post chemotherapy in a T cell acute lymphoblastic leukemia model. J Transl Med 13: 234, 2015.

15. Chou TC: Theoretical basis, experimental design, and computerized simulation of synergism and antagonism in drug combination studies. Pharmacol Rev 58: 621-681, 2006.

16. Tallarida RJ: Revisiting the isobole and related quantitative methods for assessing drug synergism. J Pharmacol Exp Ther 342: $2-8,2012$.
17. Zorzoli A, Di Carlo E, Cocco C, Ognio E, Ribatti D, Ferretti E, Dufour C, Locatelli F, Montagna D and Airoldi I: Interleukin-27 inhibits the growth of pediatric acute myeloid leukemia in NOD/SCID/Il2rg-/- mice. Clin Cancer Res 18: 1630-1640, 2012.

18. Huang C, Hu X, Wang L, Lü S, Cheng H, Song X, Wang J and Yang J: Bortezomib suppresses the growth of leukemia cells with Notch 1 overexpression in vivo and in vitro. Cancer Chemother Pharmacol 70: 801-809, 2012.

19. Witt D, Burfeind P, von Hardenberg S, Opitz L, SalinasRiester G, Bremmer F, Schweyer S, Thelen P, Neesen J and Kaulfuss S: Valproic acid inhibits the proliferation of cancer cells by re-expressing cyclin D2. Carcinogenesis 34: 1115-1124, 2013.

20. Barbetti V, Gozzini A, Cheloni G, Marzi I, Fabiani E, Santini V, Dello Sbarba P and Rovida E: Time- and residue-specific differences in histone acetylation induced by VPA and SAHA in AML1/ETO-positive leukemia cells. Epigenetics 8: 210-219, 2013.

21. Kuendgen A, Knipp S, Fox F, Strupp C, Hildebrandt B, Steidl C, Germing U, Haas R and Gattermann N: Results of a phase 2 study of valproic acid alone or in combination with all-trans retinoic acid in 75 patients with myelodysplastic syndrome and relapsed or refractory acute myeloid leukemia. Ann Hematol 84 (Suppl 1): 61-66, 2005.

22. Kuendgen A, Strupp C, Aivado M, Bernhardt A, Hildebrandt B, Haas R, Germing U and Gattermann N: Treatment of myelodysplastic syndromes with valproic acid alone or in combination with all-trans retinoic acid. Blood 104: 1266-1269, 2004.

23. Chen J, Wang G, Wang L, Kang J and Wang J: Curcumin p38-dependently enhances the anticancer activity of valproic acid in human leukemia cells. Eur J Pharm Sci 41: 210-218, 2010

24. Thomas S, Thurn KT, Biçaku E, Marchion DC and Münster PN: Addition of a histone deacetylase inhibitor redirects tamoxifentreated breast cancer cells into apoptosis, which is opposed by the induction of autophagy. Breast Cancer Res Treat 130: 437-447, 2011.

25. Sung ES, Kim A, Park JS, Chung J, Kwon MH and Kim YS: Histone deacetylase inhibitors synergistically potentiate death receptor 4-mediated apoptotic cell death of human T-cell acute lymphoblastic leukemia cells. Apoptosis 15: 1256-1269, 2010. 